\title{
Aportaciones y Limitaciones del DSM-5 desde la Psicología Clínica
}

\section{Contributions and Limitations of DSM-5 from Clinical Psychology}

\author{
Enrique Echeburúa \\ Universidad del País Vasco (UPV/EHU), España \\ Cibersam \\ Karmele Salaberría \\ Universidad del País Vasco (UPV/EHU), España \\ Marisol Cruz-Sáez \\ Universidad del País Vasco (UPV/EHU), España
}

(Rec: 13 diciembre 2013 / Acept: 03 enero 2014)

\begin{abstract}
Resumen
La reciente publicación del Manual Diagnóstico y Estadístico de los Trastornos Mentales ( $5^{a}$ edición) por la Asociación Americana de Psiquiatría ha suscitado un gran debate. Una clasificación efectiva requiere un sistema fiable y válido de los cuadros clínicos para facilitar la comunicación, elegir los tratamientos, señalar la etiología, predecir los resultados y proporcionar una base sólida para la investigación. El DSM-5 es una clasificación categorial de los trastornos mentales, pero estos no siempre encajan adecuadamente dentro de los límites de un trastorno único. Hay algunas aportaciones interesantes del DSM-5, como los capítulos de adicciones y de trastornos de la personalidad. Las adicciones ya no se limitan a las sustancias químicas, sino que se extienden a los excesos conductuales (por ejemplo, el trastorno del juego). Los trastornos de personalidad no se han modificado, pero se ha añadido un modelo alternativo en la Sección III basado en un enfoque dimensional que podría sustituir a las categorías actualmente existentes. El motivo más importante de controversia es el aumento de diagnósticos psiquiátricos, así como una exigencia menos estricta para los criterios diagnósticos en las categorías antiguamente existentes. Se comentan finalmente algunas cuestiones no resueltas con vista a investigaciones futuras.

Palabras clave: DSM-5, clasificación categorial, cambios más importantes, aportaciones, controversias.
\end{abstract}

\begin{abstract}
The recent release of the Diagnostic and Statistical Manual of Mental Disorders (5 ${ }^{\text {th }}$ edition) by the American Psychiatric Association has led to much debate. An effective classification requires a reliable and valid system for categorization of clinical phenomena in order to aid communication, select interventions, indicate aetiology, predict outcomes, and provide a basis for research. DSM-5 remains a categorical classification of separate disorders, but mental disorders do not always fit completely within the boundaries of a single disorder. There are some interesting contributions of DSM-5, such as the chapters of addictions and of personality disorders. Addiction label has also been given to behavioral excesses that have no external substance as a goal (e.g. gambling disorder). Personality disorders remain unchanged, but there is an alternative model in Section III based on a dimensional approach which might replace the current categories. The basic reason for controversy is the expansiveness of DSM-5 psychiatric diagnosis, both in terms of newly introduced categories and loosening the criteria for diagnosis in existing categories. Unanswered questions for future research in this field are commented upon.
\end{abstract}

Key words: DSM-5, categorical classification, major changes, contributions, controversies.

Correspondencia: Enrique Echeburúa. Facultad de Psicología. Universidad del País Vasco. Avda. de Tolosa, 70. 20018 San Sebastián (España). E-mail: enrique.echeburua@ehu.es

Los autores pertenecen al Grupo Consolidado de Investigación en Psicología Clínica del Gobierno Vasco y a la UFI 11/04 de la Universidad del País Vasco (UPV/EHU). 


\section{Introducción}

Los trastornos mentales son patrones de comportamiento de significación clínica que aparecen asociados a un malestar emocional o físico de la persona, a una discapacidad, al deterioro en el funcionamiento cotidiano, a la pérdida de libertad o incluso a un riesgo significativamente aumentado de implicarse en conductas contraproducentes o de morir prematuramente.

Según el estudio europeo dirigido por Wittchen et al. (2011), el 32.8\% de los europeos sufre un trastorno mental, pero solo un tercio de ellos recibe tratamiento. Asimismo, según un estudio reciente de este mismo grupo en relación con los costes (Olesen, Gustavsson, Svensson, Wittchen, Jönsson et al., 2012), los trastornos mentales (ansiedad, depresión, adicciones, trastornos de la infancia y adolescencia, etcétera) y neurológicos (tumores cerebrales, migrañas y cefaleas tensionales, trastornos del sueño, enfermedad de Parkinson, demencias, etcétera) suponen uno de los mayores retos económicos para la sanidad europea.

Por lo que a España se refiere, según la última Encuesta Nacional de Salud del Ministerio de Sanidad (Instituto Nacional de Estadística, 2006), el 21.3\% de la población adulta (un $26.8 \%$ de las mujeres y un $15.6 \%$ de los hombres) presenta riesgo de mala salud mental, que se acrecienta a medida que avanza la edad. Además, el porcentaje de pacientes en los Centros de Atención Primaria con una demanda de asistencia psicológica es de alrededor del 30\% (Gili, Roca y Serrano, 2006).

Además del aumento de los trastornos mentales, las demandas terapéuticas de la población han cambiado considerablemente en los últimos años. Ahora se tiende a consultar, además de por los cuadros clínicos "tradicionales" (depresión, trastornos de ansiedad, esquizofrenia, adicciones, entre otros), por problemas "menores", derivados de una mayor intolerancia al sufrimiento por parte de los pacientes. Estos problemas, que no constituyen propiamente trastornos mentales, reflejan una patología del sufrimiento o de la infelicidad, es decir, una situación de insatisfacción personal que no tiene en muchos casos una significación clínica. Entre ellos se encuentran el duelo por la pérdida de un ser querido, los conflictos de pareja o la ruptura de pareja no deseada, las dificultades de convivencia con los hijos adolescentes, los problemas de estrés laboral, la adaptación a nuevas situaciones en la vida o la convivencia con enfermos crónicos o incapacitados (Echeburúa, Salaberría, Corral y Cruz-Sáez, 2012).

En las clasificaciones psiquiátricas estos problemas adaptativos se denominan "códigos Z" y son un reflejo de la psicopatologización de las dificultades de la vida cotidiana, así como de los efectos de una sociedad individualista, con una carencia creciente de redes de apoyo familiar y social, que favorece la soledad y la incomunicación. El volumen de las consultas de este tipo puede suponer entre el 20\% y el $30 \%$ de las demandas asistenciales en un Centro de Salud Mental (Ortiz, González y Rodríguez, 2006).

Estas nuevas demandas terapéuticas están relacionadas con cambios sociales (entre ellos, el envejecimiento de la sociedad y los trasvases de población) y con una mayor exigencia de calidad de vida por parte de los pacientes, pero también con la medicalización de la vida diaria. De hecho, hay una tendencia errónea por asignar a todos los problemas un nombre clínico, unos síntomas y un tratamiento: es una tendencia que se ve acentuada por el marketing de la industria farmacéutica dirigido a médicos y pacientes. No deja de ser significativo, por ejemplo, que la primera edición del DSM contenía 106 trastornos mentales y que la actual (DSM-5, 2013) recoja 216" (Sandín, 2013). Con cada nueva edición los posibles trastornos mentales siempre aumentan, pero nunca se reducen. No parece razonable pensar que en el plazo de 60 años los trastornos mentales se hayan multiplicado por dos (Ezama, Alonso y Fontanil, 2010). Por ello, hay que estar precavido ante la ampliación de supuestos nuevos diagnósticos clínicos que incorpora el DSM-5: el trastorno disfórico premenstrual, el trastorno de estado de ánimo disruptivo y no regulado, el duelo patológico o el trastorno neurocognitivo leve, por citar algunos de los más llamativos.

En resumen, muchas de las consultas a los psicólogos clínicos hoy no se relacionan con trastornos mentales, sino con situaciones de infelicidad y malestar emocional. Se trata de personas que se sienten sobrepasadas en sus estrategias de afrontamiento para hacer frente a las dificultades cotidianas y que, muy frecuentemente, carecen de una red de apoyo familiar y social sólida. Los psicólogos clínicos tienen que adaptarse a esta nueva realidad, evitar la tendencia a establecer diagnósticos psiquiátricos y desarrollar unas estrategias de intervención, tales como técnicas de consejería (counselling) o de intervención en crisis, que no son exactamente las mismas que han mostrado éxito en el tratamiento de los trastornos mentales propiamente dichos. Este es un reto de futuro importante.

El objetivo de este artículo es hacer un resumen de los cambios más significativos que recoge la última versión del DSM (DSM-5) en la organización de los trastornos y en los diagnósticos específicos, así como hacer una valoración crítica de estos cambios (y, por extensión, del modelo médico de enfermedad aplicado a los trastornos mentales) desde la psicología clínica y proponer nuevas líneas de actuación. 


\section{Cambios en el DSM-5}

El DSM-5 está organizado en tres secciones. La Sección I está dirigida a proporcionar pautas para el uso clínico y forense del manual. La Sección II incluye los criterios y códigos diagnósticos de los diferentes trastornos (tabla 1) y, por último, la Sección III recoge medidas dimensionales para la evaluación de los síntomas, criterios sobre la formulación cultural de los trastornos y una propuesta alternativa sobre la conceptualización de los trastornos de personalidad, así como una descripción de las condiciones clínicas que están actualmente en estudio.

Tabla 1. Clasificación de los trastornos recogidos en el DSM-5

Trastornos del neurodesarrollo

Espectro de la esquizofrenia y otros trastornos psicóticos

Trastornos bipolares y relacionados

Trastornos depresivos

Trastornos de ansiedad

Trastornos obsesivo-compulsivos y relacionados

Trastornos relacionados con traumas y estresores

Trastornos disociativos

Trastornos de síntomas somáticos

Trastornos de la alimentación y de la conducta alimentaria

Trastornos de la eliminación

Trastornos del sueño

Disfunciones sexuales

Disforia de género

Trastornos disruptivos, del control de impulsos y conductuales

Trastornos adictivos y de consumo de sustancias

Trastornos neurocognitivos

Trastornos de la personalidad

Trastornos parafílicos

Otros trastornos

\section{Cambios en la organización de los trastornos}

A nivel estructural y organizativo, los cambios más destacables en el DSM-5 son la eliminación del sistema multiaxial y la reorganización de los capítulos. Las modificaciones habidas respecto al DSM-IV-TR figuran descritas en el Apéndice.

El DSM-5, como las ediciones anteriores de este manual, está basado en el modelo categorial, que implica que cada categoría diagnóstica es diferente de la salud y del resto de las categorías diagnósticas. Sin embargo, al ser conscientes sus autores de las limitaciones del sistema categorial, incorporan, hasta cierto punto, un enfoque más dimensional que en las ediciones anteriores. Así, por ejemplo, se analiza la severidad mayor o menor de los síntomas (leve, moderado y severo) en diversos cuadros clínicos (el trastorno depresivo mayor o los trastornos adictivos y relacionados con sustancias).
Se ha eliminado el sistema multiaxial porque generaba distinciones artificiales y era poco utilizado. Por ello, todas las categorías diagnósticas de los Ejes I y II del DSM-IVTR están incluidas en la misma sección (Sección II) en el DSM-5, con anotaciones separadas en cada categoría para las condiciones médicas asociadas (antiguo Eje III), para los factores psicosociales y ambientales (antiguo Eje IV) y para la discapacidad, entendida esta como daño en el funcionamiento social, laboral o en otras áreas significativas de la vida cotidiana (antiguo Eje V).

En la Sección III se incluyen en todos los grupos diagnósticos medidas dimensionales de la severidad o de la frecuencia en las dos últimas semanas en trece conjuntos de síntomas (doce en el caso de los niños y adolescentes), que abarcan la depresión, la ira, la manía, la ansiedad, los síntomas somáticos, la ideación suicida, la psicosis, las alteraciones del sueño, la memoria, los pensamientos y conductas repetitivas, la disociación, el funcionamiento de la personalidad y el consumo de drogas. Cada uno de estos ítems es valorado en una escala de 0 a 4 en función de su menor o mayor gravedad/frecuencia. Asimismo se incluye en esta Sección una Escala de Evaluación de la Discapacidad de la Organización Mundial de la Salud, autoadministrada, de 36 ítems, que está orientada a evaluar las capacidades del sujeto adulto para llevar a cabo actividades en seis áreas: comprensión y comunicación; movimientos; autocuidado; relación con otras personas; actividades cotidianas; y participación en la sociedad. Esta escala, que es más clara y rigurosa que la Escala de Evaluación Global del Funcionamiento del DSM-IV-TR (2000) y que cuenta también con una versión modificada para niños y adolescentes, evalúa de 1 a 5 cada una de las áreas en función de la menor o mayor dificultad que ha tenido el sujeto en los últimos 30 días.

La organización global de los capítulos (y la específica de cada capítulo) tiene más en consideración el ciclo vital que en las ediciones anteriores del DSM. Así, los cuadros clínicos que se manifiestan en las primeras fases evolutivas (como, por ejemplo, los trastornos del neurodesarrollo) figuran al principio del manual; los trastornos que habitualmente aparecen en la adolescencia y en la edad adulta joven (entre otros, los trastornos de ansiedad, depresivos o de la conducta alimentaria o los trastornos del espectro de la esquizofrenia) se encuentran descritos en la parte central; y, por último, los trastornos asociados a la vejez (trastornos neurocognitivos) se hallan al final. Asimismo en cada capítulo se describe la presentación de los cuadros clínicos, cuando es preciso, en diferentes grupos de edad (por ejemplo, en el trastorno de estrés postraumático o en los trastornos del sueño). 
Novedades relativas a los diagnósticos especificos

Los grupos diagnósticos del DSM-5 figuran descritos en la tabla 1. En este apartado, sin una pretensión de exhaustividad por razón del espacio, se comentan los cambios más significativos solo en los grupos diagnósticos más relevantes.

En el ámbito infanto-juvenil los trastornos de inicio en la infancia, la niñez o la adolescencia del DSM-IV-TR pasan a convertirse en el DSM-5 en trastornos del neurodesarrollo. El retraso mental se denomina ahora discapacidad intelectual para evitar el estigma social asociado a ese término y para hacer hincapié en la necesidad de evaluar tanto la capacidad cognitiva (cociente de inteligencia) como el grado de adaptación a la vida cotidiana. Asimismo en este capítulo los trastornos generalizados del desarrollo desaparecen como tales para integrarse en los trastornos del espectro autista, que ahora engloban el trastorno autista, el trastorno de Asperger, el trastorno desintegrativo infantil y el trastorno generalizado del desarrollo no especificado. Estos cuatro cuadros clínicos responden a una única condición con diferentes niveles de gravedad en el ámbito de la interacción social y de las conductas limitadas y repetitivas. Respecto al trastorno por déficit de atención con hiperactividad (TDAH), se ha establecido la edad anterior a los 12 años para la aparición de los síntomas (antes era a los 7 años), pero las modificaciones más importantes son la posibilidad de realizar este diagnóstico junto con un trastorno del espectro autista (antes eran incompatibles) y, especialmente, la reducción del número de síntomas para diagnosticar este cuadro clínico en la vida adulta.

En el capítulo del espectro de la esquizofrenia y otros trastornos psicóticos, el cambio más importante del DSM-5 es la desaparición de los subtipos de esquizofrenia del DSMIV-TR (paranoide, desorganizada, catatónica, indiferenciada y residual) porque han mostrado una estabilidad diagnóstica limitada y porque no han respondido diferencialmente al tratamiento (McGorry, 2010). Asimismo el calificado en el DSM-IV-TR como trastorno esquizotípico de la personalidad es considerado en el DSM-5, sin modificar los síntomas, como perteneciente al espectro de la esquizofrenia (además de estar incluido en el capítulo de los trastornos de personalidad).

El capítulo de los trastornos de ansiedad del DSM-5 supone un reagrupamiento distinto de los cuadros clínicos indicados en la edición anterior. Lo más significativo es la exclusión de este apartado del trastorno de estrés postraumático (TEPT) y del trastorno obsesivo-compulsivo (TOC), que ahora se describen en capítulos propios e independientes para resaltar el carácter distintivo de la reexperimentación del suceso traumático en el caso del TEPT o el de las obsesiones en el caso del TOC. Lo que se incluye en este capítulo son las fobias (social, específica y agorafobia), el trastorno de pánico, el trastorno de ansiedad generalizada y, además, el trastorno de ansiedad de separación y el mutismo selectivo (este último muy asociado a las respuestas de ansiedad), que figuraban en el DSM-IV-TR en el apartado de trastornos de inicio en la infancia.

El nuevo capítulo del trastorno obsesivo-compulsivo $y$ otros trastornos relacionados recoge los apartados clásicos del TOC, del trastorno dismórfico corporal y de la tricotilomanía, estos dos últimos incluidos en el apartado de trastornos de somatización y de trastornos de control de los impulsos, respectivamente, en el DSM-IV-TR, y añade algunos cuadros clínicos nuevos, como el trastorno de acumulación, que era solo un síntoma del TOC en la versión anterior, y el trastorno de excoriación (arrancarse compulsivamente la piel).

En cuanto al nuevo capítulo de los trastornos relacionados con el trauma y con el estrés, se incluyen: el TEPT, que cuenta ahora, en lugar de tres, con cuatro grupos de síntomas (reexperimentación, hiperactivación, evitación y alteraciones cognitivas/cambios en el estado de ánimo) y que tiene criterios algo distintos para los menores de 7 años; el trastorno de estrés agudo; los trastornos adaptativos, que pueden surgir ante situaciones estresantes traumáticas o no traumáticas y que antes figuraban como capítulo propio; $y$, finalmente, dos trastornos vinculados a los problemas con el apego parental y que se manifiestan en la infancia: el trastorno reactivo de la vinculación, relacionado con problemas internalizantes, y el trastorno del comportamiento social desinhibido, más vinculado con conductas temerarias e inadecuadas en las relaciones con adultos y con el TDAH.

Los trastornos depresivos y los trastornos bipolares se agrupan en capítulos distintos. El capítulo de los trastornos depresivos incluye, además del trastorno depresivo mayor y de la distimia, nuevos cuadros clínicos, como el trastorno disfórico premenstrual o el trastorno del estado de ánimo disruptivo y no regulado, este último orientado a niños y adolescentes (hasta los 18 años) con síntomas persistentes de irritabilidad y episodios de descontrol conductual y que tiene el objetivo de evitar un sobrediagnóstico del trastorno bipolar en la infancia. A su vez, el duelo no excluye el diagnóstico de un episodio depresivo mayor. El DSM-IV-TR excluía de este diagnóstico a las personas que mostraban dichos síntomas tras la pérdida de un ser querido en los dos meses anteriores, pero el DSM-5 omite esta exclusión.

En el capítulo de los trastornos de la alimentación y de la conducta alimentaria, además de la anorexia nerviosa y de 
la bulimia nerviosa, se integran la pica y la rumiación (antes incluidos en los trastornos de la infancia y adolescencia) y se incluyen en el DSM-5 como nuevos diagnósticos el trastorno evitativo/restrictivo de la ingesta y el trastorno por atracón, que aporta como elemento diferencial de la bulimia la ausencia de implicación en conductas compensatorias inadecuadas (purgarse, hacer ejercicio excesivo e irracional, etcétera). En el apartado de la anorexia nerviosa ya no se requiere la amenorrea como criterio para el diagnóstico porque hay situaciones en que esta circunstancia no aparece (mujeres tomando anticonceptivos o que han llegado a la menopuasia, hombres afectados por este cuadro clínico, etcétera).

El capítulo del DSM-IV-TR referido a los trastornos relacionados con sustancias se amplía en el DSM-5 y se denomina trastornos adictivos y relacionados con sustancias. Es más preciso hablar de adicción que de dependencia porque la tolerancia y el síndrome de abstinencia que caracterizan a la dependencia son respuestas que están también presentes como respuesta a algunos psicofármacos (benzodiacepinas, por ejemplo) y que no necesariamente indican la presencia de una adicción, que requiere además el ansia por una droga o por una conducta placentera. De este modo, a las adicciones químicas se añade el trastorno del juego (antes denominado juego patológico e incluido en el DSM-IV-TR en el capítulo de trastornos del control de los impulsos). Asimismo se abre el campo a futuros nuevos diagnósticos (adicción a Internet o adicción al sexo, por ejemplo). El núcleo de este apartado es la adicción en sí misma (el ansia por la droga/conducta placentera se ha incorporado como criterio diagnóstico), independientemente de que esta sea generada por drogas o por conductas placenteras, porque la sintomatología es similar y porque la activación del sistema de recompensa cerebral es muy parecido. Asimismo la distinción entre dependencia y abuso desaparece y se toma en consideración el trastorno por uso de sustancias, con diversos grados de gravedad (leve, moderado y severo).

Respecto al nuevo capítulo de los trastornos neurocognitivos, el DSM-5 recoge las demencias y los trastornos amnésicos del DSM-IV-TR, pero incluye además un apartado específico (y nuevo) para el trastorno neurocognitivo leve, que supone un deterioro de las funciones cognitivas que no es meramente atribuible a la edad y que interfiere negativamente en la vida cotidiana de la persona. Asimismo se especifican los trastornos neurocognitivos, ya sean severos o leves, en función de subtipos etiológicos más amplios que en el DSM-IV-TR (enfermedad de Alzheimer, demencia vascular, demencia frontotemporal, demencia de cuerpos de Lewy, lesión traumática cerebral, enfermedad de
Parkinson, infección por VIH, enfermedad de Huntington y encefalopatía espongiforme).

El capítulo de los trastornos sexuales y de la identidad sexual del DSM-IV-TR se desdobla en tres capítulos distintos en el DSM-5 (disfunciones sexuales, disforia de género y trastornos parafílicos). Las disfunciones sexuales ya no se clasifican en función del ciclo de la respuesta sexual, porque la distinción entre ciertas fases (por ejemplo, deseo y activación) puede ser artificial, sino de forma más descriptiva y más exigente en cuanto a la duración y la gravedad del trastorno. Asimismo desaparece el trastorno de aversión al sexo por su infrecuencia y se fusionan la dispareunia y el vaginismo en el trastorno de penetración/dolor genital. A su vez, el capítulo de la disforia de género, que pone el énfasis en la "incongruencia de género", es nuevo en el DSM-5 y sustituye al trastorno de la identidad sexual del DSM-IV-TR. Por último, en el capítulo de los trastornos parafilicos se establece una distinción entre las parafilias y los trastornos parafílicos, que son parafilias que causan malestar a la persona afectada o cuya satisfacción implica daño a terceras personas. Es decir, la novedad del DSM-5 es que la parafilia es una condición necesaria, pero no suficiente, para diagnosticar un trastorno parafílico.

Asimismo hay en el DSM-5 un nuevo capítulo (trastornos del control de los impulsos, disruptivos y de conducta) que supone una integración de dos capítulos del DSM-IV-TR y que abarca los trastornos caracterizados por problemas en el autocontrol emocional y conductual. Del antiguo capítulo de los trastornos del control de los impulsos se recogen ahora el trastorno explosivo intermitente, la piromanía y la cleptomanía; y del capítulo de los trastornos de la infancia y adolescencia se incluyen ahora el trastorno negativista desafiante, el trastorno de conducta y el trastorno de conductas disruptivas. Asimismo el trastorno antisocial de la personalidad se incluye tanto en este capítulo como en el referido a los trastornos de personalidad. Respecto al trastorno negativista desafiante, se agrupan los criterios en tres bloques (estado de ánimo de enfado o irritable, conductas desafiantes y actitudes de venganza) para abarcar síntomas tanto cognitivos como conductuales y se especifican criterios de frecuencia y severidad de los síntomas. En el DSM-5 el trastorno explosivo intermitente incluye entre los arrebatos violentos, no solo la agresividad física, sino también la violencia verbal y especifica los criterios de frecuencia precisos para hacer el diagnóstico.

Mención aparte merece el capítulo de los trastornos de personalidad (TP). La clasificación y el criterio categorial utilizados en el DSM-5 son los mismos que en el DSM-IVTR, excepto que ya no figuran en el Eje II. De este modo, se 
señalan tres clusters que agrupan los diez trastornos específicos de personalidad: a) cluster A (trastornos paranoide, esquizoide y esquizotípico), vinculado a personas con conductas extravagantes y excéntricas; b) cluster $\mathrm{B}$ (trastornos antisocial, límite, histriónico y narcisista), relacionado con personas con conductas dramáticas, emocionales o erráticas; y c) cluster C (evitativo, dependiente y obsesivo-compulsivo), referido a personas con conductas de ansiedad.

Sin embargo, en la Sección III del DSM-5 se presenta adicionalmente un modelo alternativo para los TP, que está basado en el daño causado por el funcionamiento de la personalidad y en los rasgos de personalidad patológicos. Este enfoque agrupa un número de TP más restrictivo (antisocial, evitativo, límite, narcisista, obsesivo-compulsivo y esquizotípico) que en el enfoque categorial, así como un trastorno de personalidad-rasgo, referido a la presencia de un trastorno de personalidad que no encaja en los criterios diagnósticos para los trastornos específicos y que sustituye al trastorno de la personalidad no especificado del DSM-IV-TR.

\section{Insuficiencias del DSM-5}

En el DSM-5, como en sus ediciones anteriores, se habla de trastornos mentales, no de enfermedades mentales. El concepto de enfermedad implica una etiología, una agrupación de síntomas, un curso y un pronóstico, así como una determinada respuesta al tratamiento. Sin embargo, hoy no es posible determinar los factores etiológicos ni los procesos patológicos subyacentes para la mayoría de los cuadros clínicos. Por ello, se opta por limitarse a describir de la forma más detallada posible los criterios diagnósticos actualizados de los trastornos mentales para que puedan ser identificados por los terapeutas y ser comunicados a los profesionales con un lenguaje común. En este sentido el DSM-5, como sus antecesores, es más un diccionario descriptivo que un manual de psicopatología.

Sin embargo, el diagnóstico resultante del proceso de evaluación psicológica debe ir más allá de la etiquetación de los problemas. Es decir, además de responder a la pregunta de ¿qué le pasa al paciente?, la evaluación debe responder a la pregunta de ¿por qué le pasa esto al paciente?, lo que requiere, por un lado, del análisis topográfico y, por otro, del análisis funcional de la conducta. La etiquetación de los problemas del paciente es solo un paso en el proceso terapéutico.

Aun siendo el DSM el principal referente en la salud mental mundial, con los años y tras sucesivas revisiones se ha empezado a cuestionar seriamente su validez, basada fundamentalmente en el consenso más que en las pruebas científicas. El DSM-5 se entronca en el modelo médico categorial de enfermedad, que, en el caso de los trastornos mentales, presenta muchas limitaciones en la práctica clínica. Así, por ejemplo, hay grupos de síntomas, relacionados especialmente con la ansiedad y la depresión, que están presentes en muchas categorías diagnósticas. De hecho, los límites entre las categorías diagnósticas son mucho más permeables de lo que figura en el texto. Un ejemplo es el trastorno mixto de ansiedad y depresión, que no está incluido en el manual y que, sin embargo, es uno de los trastornos con mayor tasa de prevalencia en asistencia primaria (London School of Economics, 2006). Asimismo muchos pacientes con un mismo diagnóstico presentan perfiles sintomáticos muy variados, por lo que el establecimiento de un diagnóstico clínico no es siempre clarificador del tratamiento a seguir.

Es más, la casi inexistencia de pacientes con un único diagnóstico puro (la comorbilidad es la norma, no la excepción), el recurso a los diagnósticos no especificados, que pueden ser los más frecuentes en áreas como los trastornos de la conducta alimentaria, los trastornos de personalidad o los trastornos del espectro autista, o la falta de tratamientos específicos para muchas categorías diagnósticas revela las imprecisiones de este sistema clasificatorio (Echeburúa, Salaberría, Corral y Polo-López, 2010).

En concreto, hay nuevos trastornos diagnosticados en el DSM-5 que no cuentan con un apoyo empírico sólido y que pueden implicar una medicalización de conductas normales, con el consiguiente riesgo de medicación innecesaria: a) el trastorno del estado de ánimo disruptivo y no regulado, que puede ser una variante de las rabietas temperamentales en niños y adolescentes; b) el trastorno de la comunicación social-pragmática; c) el duelo por la pérdida de un ser querido como un posible trastorno depresivo mayor, que puede implicar la psicopatologización de un sufrimiento normal en esas circunstancias; d) el trastorno neurocognitivo leve, que puede ser reflejo de la pérdida de memoria atribuible a la edad y que no es necesariamente precursora de demencia; e) la extensión del diagnóstico de trastorno por déficit de atención con hiperactividad a la vida adulta, con el posible aumento de la prescripción de drogas estimulantes; e) el trastorno por atracón, cuya definición puede resultar imprecisa ("comida excesiva 12 veces en el plazo de 3 meses") para diferenciarla de la simple glotonería; y f) las molestias y el malestar emocional periódico de la menstruación como el trastorno disfórico menstrual (Frances, 2013).

Respecto a los trastornos de personalidad, no se ha dado el paso decidido a un enfoque dimensional de los TP. Se mantiene el criterio categorial del DSM-IV-TR, si bien 
se ha propuesto en la Sección III un modelo alternativo híbrido como guía para la investigación futura y que incluye la evaluación del funcionamiento de la personalidad y de los rasgos de personalidad patológicos en seis TP. El enfoque categorial de los trastornos de personalidad presenta muchas limitaciones, tales como la comorbilidad frecuentemente registrada entre diversos TP o la presencia de características de más de un trastorno específico de la personalidad que no cumplen los criterios completos para ningún trastorno concreto y que llevan con frecuencia al diagnóstico de trastorno de personalidad no especificado (Esbec y Echeburúa, 2011). Por ello, el modelo alternativo presentado en la Sección III del DSM-5, basado en el funcionamiento de la personalidad y en criterios basados en rasgos, entronca más con la investigación en psicología de la personalidad y dota a los trastornos descritos de una mayor fundamentación empírica. En cuanto a los trastornos específicos, puede resultar chocante que se mantenga en el DSM-5 el trastorno antisocial de la personalidad cuando hay una investigación sólida que respalda la existencia de un TP más específico y preciso denominado psicopatía, que abarca dos factores: los componentes antisociales, recogidos en el DSM-5, y los componentes afectivos de este trastorno (falta de empatía, crueldad, ausencia de remordimiento) (Esbec y Echeburúa, 2010, 2011; Echeburúa y Fernández-Montalvo, 2007; Fernández-Montalvo y Echeburúa, 2008).

En último término, las insuficiencias del DSM-5 derivan del modelo médico de enfermedad mental. El sufrimiento humano es el resultado de una compleja combinación de factores biológicos, psicológicos y sociales, lo que implica la necesidad de la formulación psicológica y el necesario conocimiento de las historias de vida de los pacientes (en lugar o además del diagnóstico psiquiátrico), es decir, de una evaluación y de un tratamiento psicológico individualizado, fundamentado en las terapias psicológicas basadas en la evidencia (Echeburúa et al., 2010).

\section{Conclusiones}

Contar con diagnósticos clínicos válidos y fiables debería ser esencial para predecir el curso del trastorno, enfocar adecuadamente el tratamiento, evaluar los resultados de la terapia, calcular las tasas de prevalencia de los diferentes trastornos mentales a efectos de planificar los servicios asistenciales o identificar correctamente a los pacientes para las investigaciones clínicas. Sin embargo, los diagnósticos clínicos solo deben emplearse cuando están basados en pruebas científicas y muestran una utilidad clínica (Frances, 2013; Kraemer, Kupfer, Clarke, Narrow y Regier, 2012).

Las nosologías psiquiátricas tienen diversas ventajas, como son facilitar la comunicación entre profesionales, utilizando un lenguaje común, y unificar los criterios de investigación clínica en los diferentes países. De las dos clasificaciones más difundidas (la CIE-10, de la Organización Mundial de la Salud, y el DSM-5), cada vez más próximas entre sí en sus últimas ediciones, esta última es la más influyente porque cuenta con el respaldo de la poderosa Asociación Americana de Psiquiatría y porque es más operativa (los criterios diagnósticos son concisos y explícitos) y menos narrativa que la CIE-10. De este modo, los criterios diagnósticos para la selección y evaluación de los pacientes en los ensayos clínicos publicados en las principales revistas están basados en el DSM, lo que supone una influencia directa para los investigadores clínicos. Por ello, va a ser un referente (o incluso un vademécum) en la investigación en psiquiatría y psicología clínica en los próximos años (Nemeroff et al., 2013).

Sin embargo, el abuso de los diagnósticos psiquiátricos o la ampliación de categorías diagnósticas recogida en el DSM-5, así como el requerimiento de umbrales diagnósticos menos exigentes para muchos trastornos, puede traer consigo la psicopatologización de algunos problemas de la vida cotidiana, la generación de pacientes falsos positivos, la estigmatización del paciente asociada a los diagnósticos psiquiátricos y al uso generalizado e indiscriminado de la prescripción farmacológica, lo que constituye un fenómeno especialmente preocupante en el ámbito infantil (Boyle, 2013; Carlat, 2010). Es decir, muchas personas normales con problemas de duelo, comida excesiva, distraibilidad, reacciones al estrés, olvidos en la vejez o rabietas infantiles pueden quedar atrapadas en la red de este manual diagnóstico (Frances, 2013; Wakefield y First, 2012). Parece olvidarse de que existen muchos modos y maneras de ser normal. La tolerancia hacia comportamientos diferentes y hacia la diversidad cultural es importante, así como lo es tener en cuenta que el sufrimiento es inherente a la vida. Ningún comportamiento, sentimiento o actividad mental puede calificarse de patológico sin examinar su posible utilidad adaptativa y estratégica y las condiciones contextuales en las que aparece.

La tendencia a crear cada vez más etiquetas diagnósticas para describir comportamientos específicos, en lugar de agrupar los síntomas de los pacientes en categorías amplias, va a conducir al absurdo de que un paciente tenga muchas etiquetas asignadas. Además, la incapacidad para conectar los diagnósticos con los procesos causales lleva a un callejón sin salida. 
Ello puede ser así al margen de que una cautela razonable del DSM-5 es que la identificación de un trastorno requiere en todos los casos que los síntomas causen un malestar clínico significativo o una interferencia grave en la vida cotidiana del sujeto afectado y que se recomiende contar con información adicional procedente de los familiares o de otras personas allegadas al paciente (Barker, 2011).

La tendencia al sobrediagnóstico clínico constituye un error en la práctica profesional. A veces hay problemas que interfieren negativamente en la vida de una persona y que desbordan sus recursos de afrontamiento, pero que no constituyen propiamente un trastorno mental. Por ello, se puede ayudar y tratar psicológicamente a una persona que tiene problemas, incluso síntomas clínicos, pero que no llegan a ser un trastorno: el tratamiento no requiere necesariamente el etiquetaje diagnóstico de un problema (Bracken et al., 2012; British Psychological Society, 2011; Echeburúa, 2010).
Un reto de futuro es integrar el sistema categorial, que está basado en la entrevista clínica, con el enfoque dimensional, que se apoya fundamentalmente en el autoinforme del sujeto. Todo ello puede ser potenciado si se encuentran en el futuro marcadores psicológicos o biológicos de la enfermedad basados en las pruebas de neuroimagen, en la evaluación neuropsicológica, en los test de laboratorio, en la epidemiología o en la genética (Szyf y Bick, 2013).

Por último, se requiere una sólida formación clínica para establecer un diagnóstico en el DSM-5 porque los criterios establecidos se refieren a síntomas, conductas, funciones cognitivas, signos físicos y combinaciones de síntomas que es preciso discriminar con precisión de lo que son los cambios en la vida cotidiana, la adaptación a las diversas fases del ciclo evolutivo o las respuestas transitorias a las situaciones de estrés.

Apéndice. Cambios más importantes del DSM-IV-TR al DSM-5

\begin{tabular}{ll}
\hline \multicolumn{1}{c}{ DSM-IV-TR } & \multicolumn{1}{c}{ DSM-5 } \\
\hline Trastornos de inicio en la infancia, niñez o adolescencia & Trastornos del neurodesarrollo \\
- Retraso mental & - Discapacidad intelectual \\
- Trastornos del aprendizaje & Trastorno específico del aprendizaje \\
- Trastorno de las habilidades motoras & - Trastornos motores \\
- Trastornos de la comunicación & - Trastornos de la comunicación \\
- Trastornos generalizados del desarrollo & - Trastorno del espectro autista \\
- Trastornos por déficit de atención y comportamiento perturbador & - Trastorno por déficit de atención e hiperactividad \\
- Trastornos de la ingestión y de la conducta alimentaria de la infancia & \\
o la niñez & \\
- Trastornos de tics & \\
- Trastornos de la eliminación & \\
- Otros trastornos de la infancia, niñez o adolescencia: ansiedad de \\
separación, mutismo selectivo, trastorno reactivo de la vinculación, \\
trastorno de movimientos estereotipados
\end{tabular}

Trastornos neurocognitivos

Delirium, demencia, trastorno amnésico y otros trastornos cognoscitivos • Delirium

- Trastornos neurocognitivos severos y leves

Trastornos mentales debidos a enfermedad

Trastornos relacionados con sustancias

- Trastornos por consumo de sustancias (dependencia y abuso)

- Trastornos inducidos por sustancias (intoxicación y abstinencia)

Alcohol, alucinógenos, anfetaminas, cafeína, cannabis, cocaína, fenciclidina, inhalantes, nicotina, opiáceos, sedantes, hipnóticos y ansiolíticos

Esquizofrenia y otros trastornos psicóticos

\section{Trastornos del estado de ánimo}

Espectro de la esquizofrenia y otros trastornos psicóticos

\section{Otros trastornos mentales}

Trastornos adictivos y relacionados con sustancias

- Alcohol, cafeína, cannabis, alucinógenos, inhalantes, opiáceos, sedantes, hipnóticos y ansiolíticos, estimulantes, tabaco.

- Trastornos no relacionados con sustancias: trastorno del juego Trastornos bipolares y relacionados

Trastornos depresivos 
Trastornos de ansiedad

Trastorno obsesivo-compulsivo y otros trastornos relacionados

Trastornos de ansiedad

Trastornos relacionados con el trauma y con el estrés

- Trastorno de estrés agudo

- Trastorno de estrés postraumático

- Trastornos adaptativos

- Trastorno reactivo de la vinculación

- Trastorno del comportamiento social desinhibido

Trastornos con síntomas somáticos y relacionados

- Trastorno con síntomas somáticos

Trastornos somatomorfos

- Ansiedad por la enfermedad

- Trastorno de conversión

- Factores psicológicos que afectan a condiciones médicas

- Trastornos facticios

\begin{tabular}{l}
\hline Trastornos facticios \\
\hline Trastornos disociativos \\
\hline Trastornos sexuales y de la identidad sex \\
- Trastornos sexuales \\
- Parafilias \\
- Trastornos de la identidad sexual \\
\hline Trastornos de la conducta alimentaria \\
- Anorexia \\
- Bulimia
\end{tabular}

Trastornos del sueño

Trastornos del control de los impulsos no clasificados en otros apartados

- Trastorno explosivo intermitente

- Cleptomanía

- Piromanía

- Tricotilomanía

- Juego patológico

\section{Trastornos disociativos}

Disfunciones sexuales

Trastornos parafílicos

Disforia de género

Trastornos de alimentación y de la conducta alimentaria

- Anorexia

- Bulimia

- Pica

- Rumiación

- Trastorno evitativo/restrictivo de la ingesta

- Trastorno por atracón
Trastornos del sueño

Trastornos del control de los impulsos, disruptivos y de conducta

- Trastorno explosivo intermitente

- Cleptomanía

- Piromanía

- Trastorno negativista desafiante

- Trastorno de conducta

- Trastorno de personalidad antisocial

Trastornos adaptativos

Trastornos de eliminación

- Enuresis

- Encopresis

Trastornos de personalidad

- Trastornos de la personalidad del grupo A

- Trastornos de la personalidad del grupo B

- Trastornos de la personalidad del grupo C

\section{Trastornos de personalidad}

- Trastornos de la personalidad del grupo A

- Trastornos de la personalidad del grupo B

- Trastornos de la personalidad del grupo C

\section{Referencias}

American Psychiatric Association (1952). Diagnostic and statistical manual: Mental disorders. Washington, DC: Author.

American Psychiatric Association (2000). DSM-IV-TR. Diagnostic and statistical manual of mental disorders ( $4^{\text {th }}$ Edition Reviewed). Washington, DC: Author.
American Psychiatric Association (2013). DSM-5. Diagnostic and statistical manual of mental disorders ( $5^{\text {th }}$ Edition). Washington, DC: Author. Barker, P. (2011). Psychiatric diagnosis. En P. Barker (Ed.), Mental health ethics: The human context (pp. 139-148). Abingdon, NY: Routledge.

Boyle, M. (2013). The persistence of medicalization: Is presentation of alternatives part of the problema? En S. Coles, S. Keenan y B. Diamond(Eds.), Madness contested: Power and practice (pp. 3-22). Ross-on-Wye: PCCS Books. 
Bracken, P., Thomas, P., Timimi, S., Asen, E., Behr, G., ... Beuster, C. (2012). Psychiatry beyond the current paradigm. Bristish Journal of Psychiatry, 201, 430-434.

British Psychological Society (2011). Response to the American Psychiatric Association: DSM-5 development. Leicester: British Psychological Society.

Carlat, D. (2010). Unhinged: The Trouble with Psychiatry - A Doctor's Revelations about a Profession in Crisis. New York: Free Press.

Echeburúa, E. (2010). The challenge of posttraumatic stress disorder prevention: how to survive a disaster? Terapia Psicológica, 28, 147-154.

Echeburúa, E., y Fernández-Montalvo, J. (2007). Male batterers with and without psychopathy: An exploratory study in Spanish prisons. International Journal of Offender Therapy and Comparative Criminology, 51, 254-263.

Echeburúa, E., Salaberría, K., Corral, P., y Cruz-Sáez, M.S. (2012). Funciones y ámbitos de actuación del psicólogo clínico y del psicólogo general sanitario: una primera reflexión. Behavioral Psychology/Psicología Conductual, 20, 423-435.

Echeburúa, E., Salaberría, K., Corral, P., y Polo-López, R. (2010). Terapias psicológicas basadas en la evidencia: limitaciones y retos de futuro. Revista Argentina de Clínica Psicológica, 19, 247-256.

Esbec, E., y Echeburúa, E. (2010). Violencia y trastornos de la personalidad: implicaciones clínicas y forenses. Actas Españolas de Psiquiatría, 38, 249-261.

Esbec, E., y Echeburúa, E. (2011). La reformulación de los trastornos de la personalidad en el DSM-V. Actas Españolas de Psiquiatría, 39, 1-11.

Ezama, E., Alonso, Y., y Fontanil, Y. (2010). Pacientes, síntomas, trastornos, organicidad y psicopatología. International Journal of Psychology and Psychological Therapy, 10, 293-314.

Fernández-Montalvo, J., y Echeburúa, E. (2008). Trastornos de personalidad y psicopatía en hombres condenados por violencia grave contra la pareja. Psicothema, 20, 193-198.

Frances, A. (2013). Saving Normal: An Insider's Revolt Against Out-ofControl Psychiatric Diagnosis, DSM-5, Big Pharma, and the Medicalization of Ordinary Life. London: William Morrow (HarperCollins Publishers).
Gili, M., Roca, M., y Serrano, M. J. (2006). Trastornos mentales e hiperfrecuentación en atención primaria. INFOCOP. Recuperado desde http: //infocop.es/view-article.asp?id=1025.

Instituto Nacional de Estadística (2006). Encuesta Nacional de Salud. Recuperado desde http://www.ine.es/metodologia/t15/t1530419cues06.htm.

Kraemer, H. C., Kupfer, D. J., Clarke, D. E., Narrow, W. E., y Regier, D. A. (2012). DSM-5: how reliable is reliable enough? American Journal of Psychiatry, 169, 13-15.

London School of Economics (2006). The Depression Report. A new deal for depression and anxiety disorders. London: The Centre for Economic Performance's Mental Health Policy Group.

McGorry, P. D. (2010). Risk syndromes, clinical staging and DSM-V: new diagnostic infrastructure for early intervention in psychiatry. Schizophrenia Research, 120, 49-53.

Nemeroff, C. B., Weinberger, D., Rutter, M., MacMillan, H. L., Bryant, R. A., ...Wessely, S. (2013). DSM-5: a collection of psychiatrist views on the changes, controversies, and future directions. BMC Medicine, 11, 202.

Olesen, J., Gustavsson, A., Svensson, M., Wittchen, H. U., y Jönsson, B (2012). On behalf of the CDBE2010 Study Group and the European Brain Council The economic cost of brain disorders in Europe. European Journal of Neurology, 19, 155-162.

Ortiz, A., González, R., y Rodríguez, F. (2006). La derivación a salud mental de pacientes sin un trastorno psíquico diagnosticable. Atención Primaria, 38, 563-569.

Sandín, B. (2013). DSM-5: ¿Cambio de paradigma en la clasificación de los trastornos mentales? Revista de Psicopatología y Psicología Clínica, 18, 255-286.

Szyf, M., y Bick, J. (2013). DNA methylation: A mechanism for embedding early life experiences in the genome. Child Development, 84, 49-57.

Wakefield, J. C., y First, M.B. (2012). Does the empirical evidence support the proposal to eliminate the major depression "bereavement exclusion" in DSM-5? World Psychiatry, 11, 3-10.

Wittchen, H. U., Jacobi, F., Rehm, J., Gustavsson, A., Svensson, M., Jönsson, B., ... (2011). The size and burden of mental disorders and other disorders of the brain in Europe 2010. European Neuropsychopharmacology, 21, 655-679. 